\title{
Evaluating the Impact of a Collaborative Care Model in Diabetes Management in a Primary Healthcare Setting in Qatar Using Real-World Data
}

Sara Abdulrhim¹, Ahmed Awaisu'1, Mohamed Izham¹, Mohammed Diab¹, Mohamed Abdelazim², Hend Al-Raey², Mohammed Thahir², Sownd Sankaralingam"

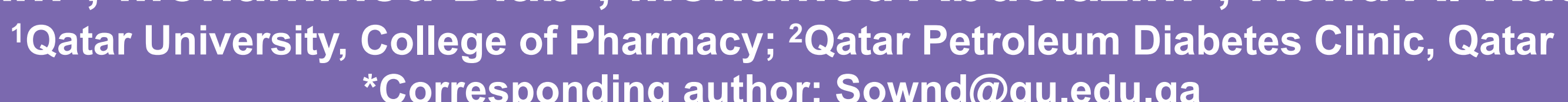

\section{Background}

Diabetes is a chronic, debilitating illness of an escalating prevalence worldwide ${ }^{1}$

The current prevalence of diabetes in Qatar is $15.5 \%$ which is projected to increase to $29.7 \%$ by $2035^{2}$.

Diabetes management is still challenging despite healthcare advancement, warranting the need for a comprehensive Collaborative Care Model (CCM)

- CCM assures the integration of knowledge, skills, values and attitudes that aid team working within and among professions, and with patients and their families to improve health outcomes ${ }^{4}$.

- Therefore, we aim to evaluate the impact of CCM on diabetes-related outcomes at a primary healthcare setting in Qatar.

\section{Objectives}

I. To characterize the clinical profile including diabetesrelated comorbidities and complications of patients with DM attending an ambulatory diabetes care clinic at a primary healthcare center.

II. To evaluate the impact of CCM on glycemic control [glycated hemoglobin $A_{1 c}\left(H_{b A_{1 c}}\right)$, fasting plasma glucose (FBG), and random blood glucose (RBG)] among these patients.

III. To evaluate the impact of the CCM on other diseaserelated outcomes comprising lipid profile, BP, and body mass index (BMI).

\section{Methods}

- Study design: Retrospective observational study

- Outcome measures: $\mathrm{HbA}_{1 \mathrm{c}}, \mathrm{FBG}, \mathrm{BMI}$, blood pressure (BP), and lipid profile.

- Statistical analysis: Descriptive and inferential statistics.
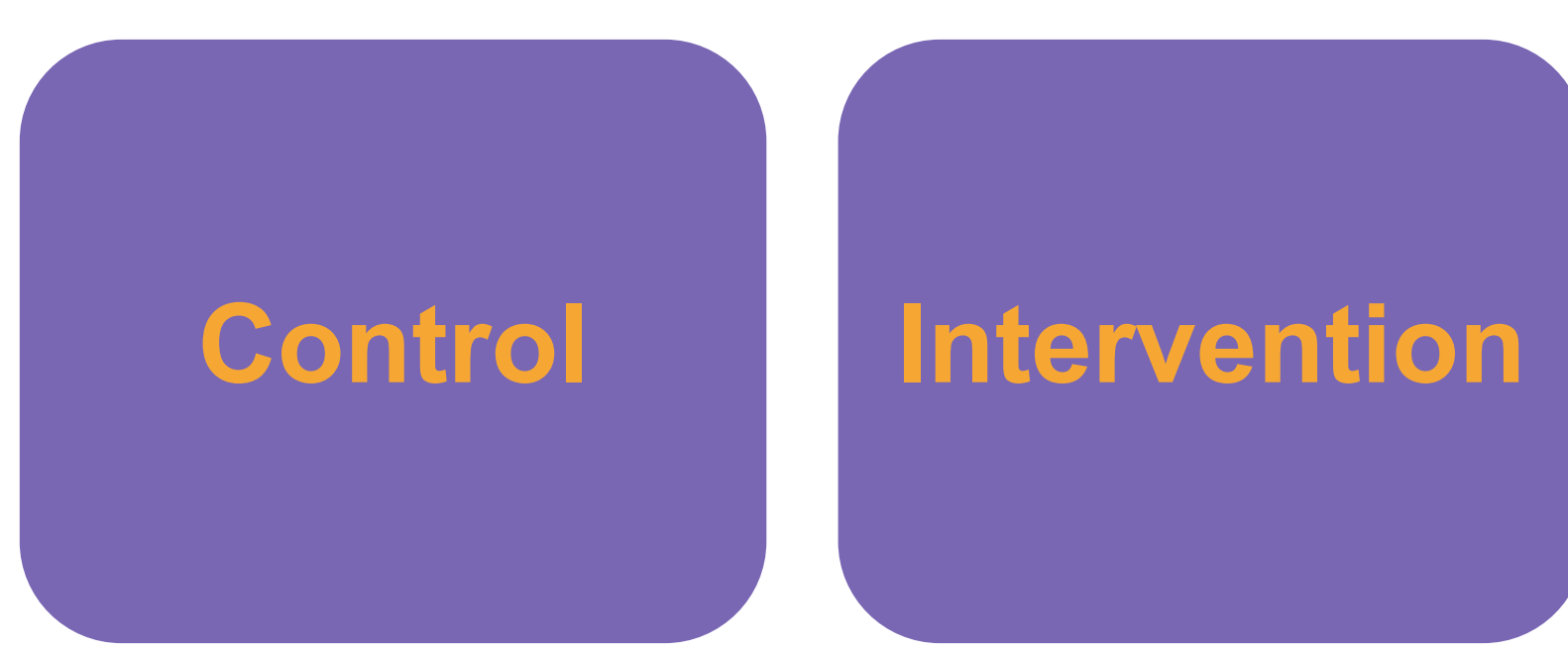

Baseline

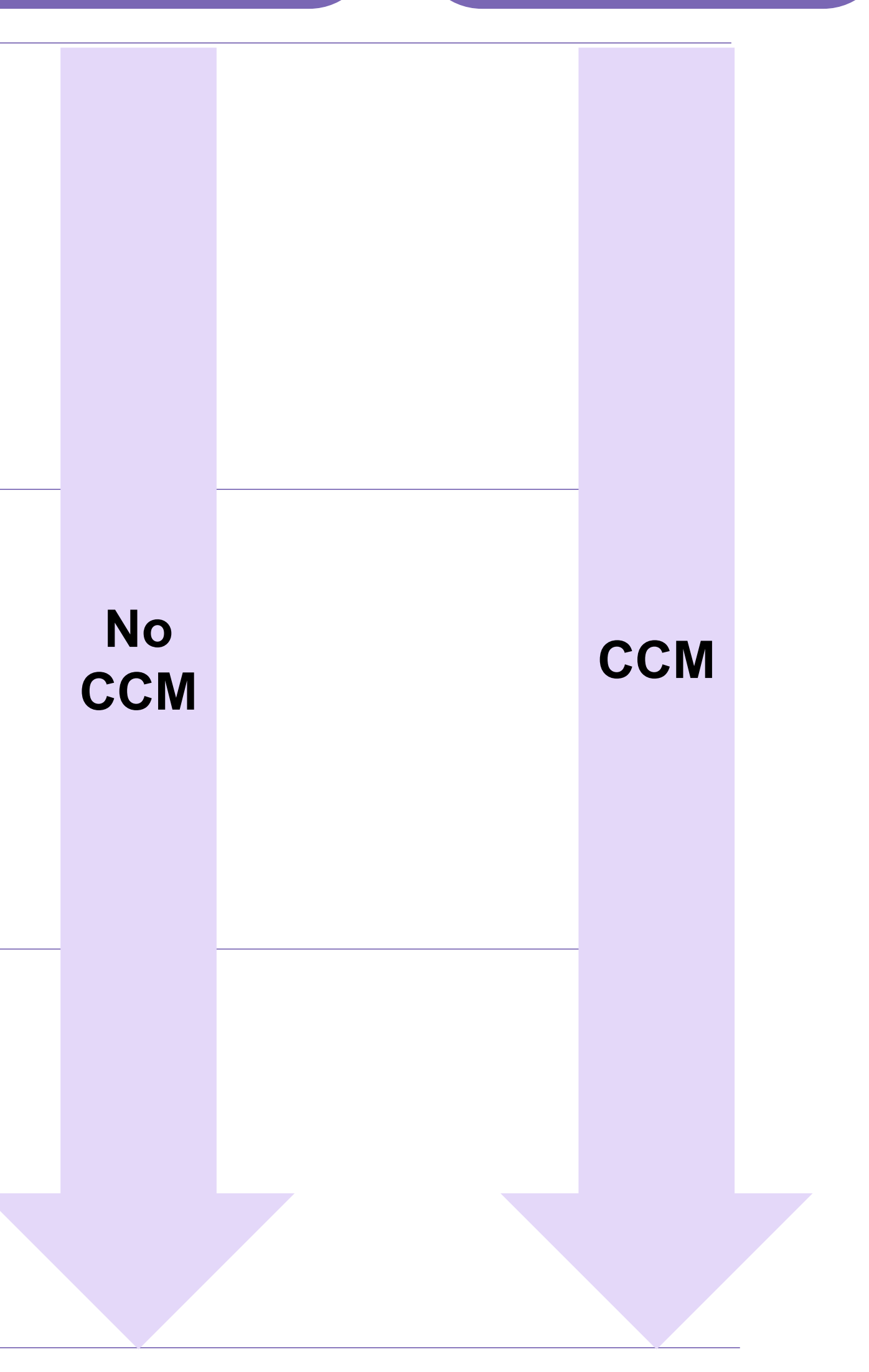

\section{Results}

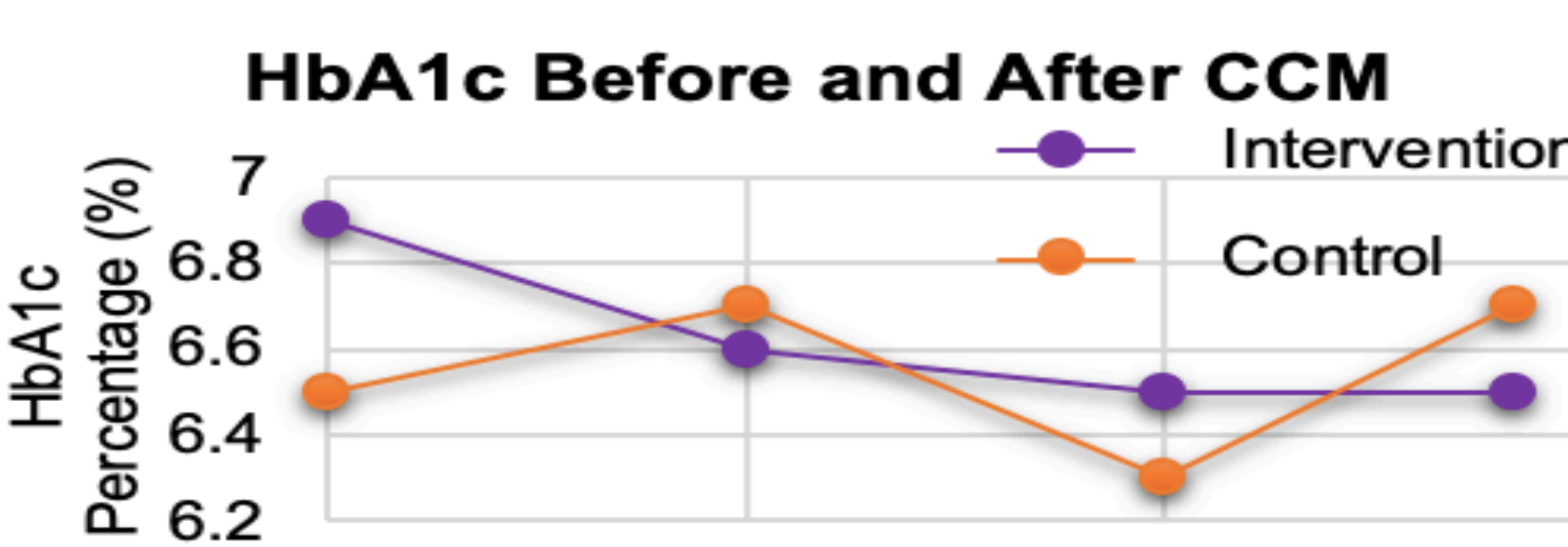

RBG Before and After CCM

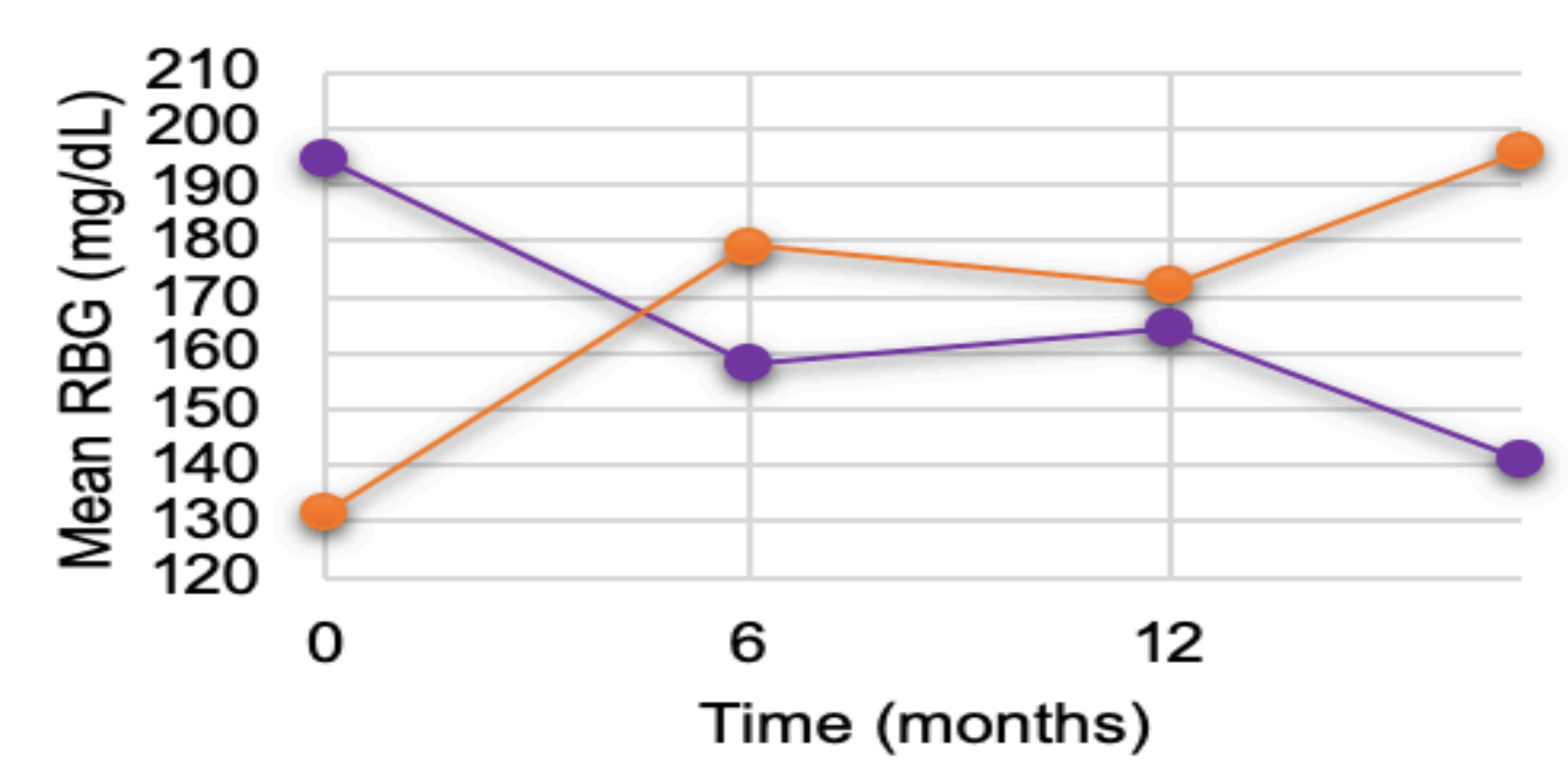

Figure 1. Glycemic control parameters

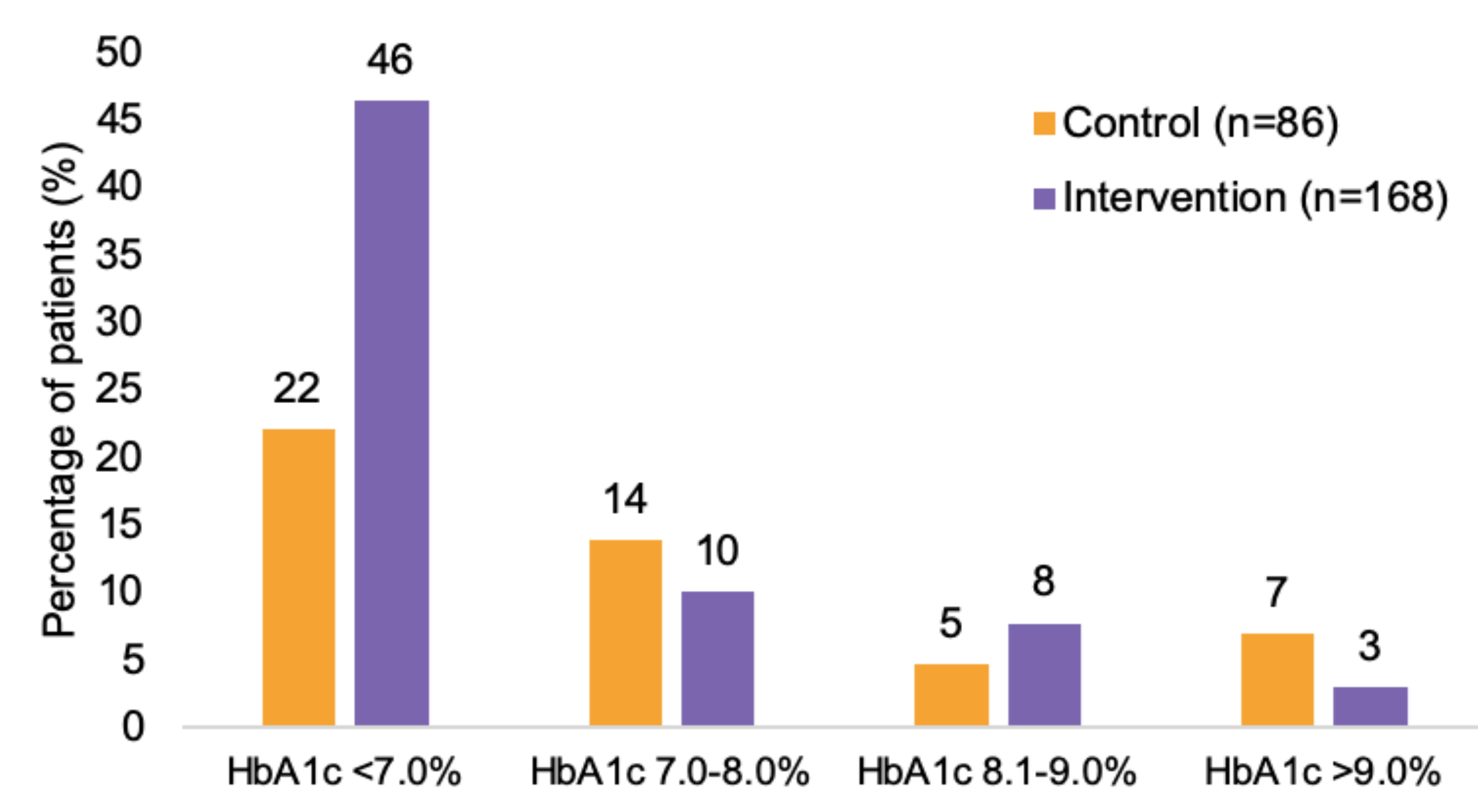

Figure 2. Final mean $\mathrm{HbA} 1 \mathrm{C}$ distribution at 17 months

LDL Before and After CCM
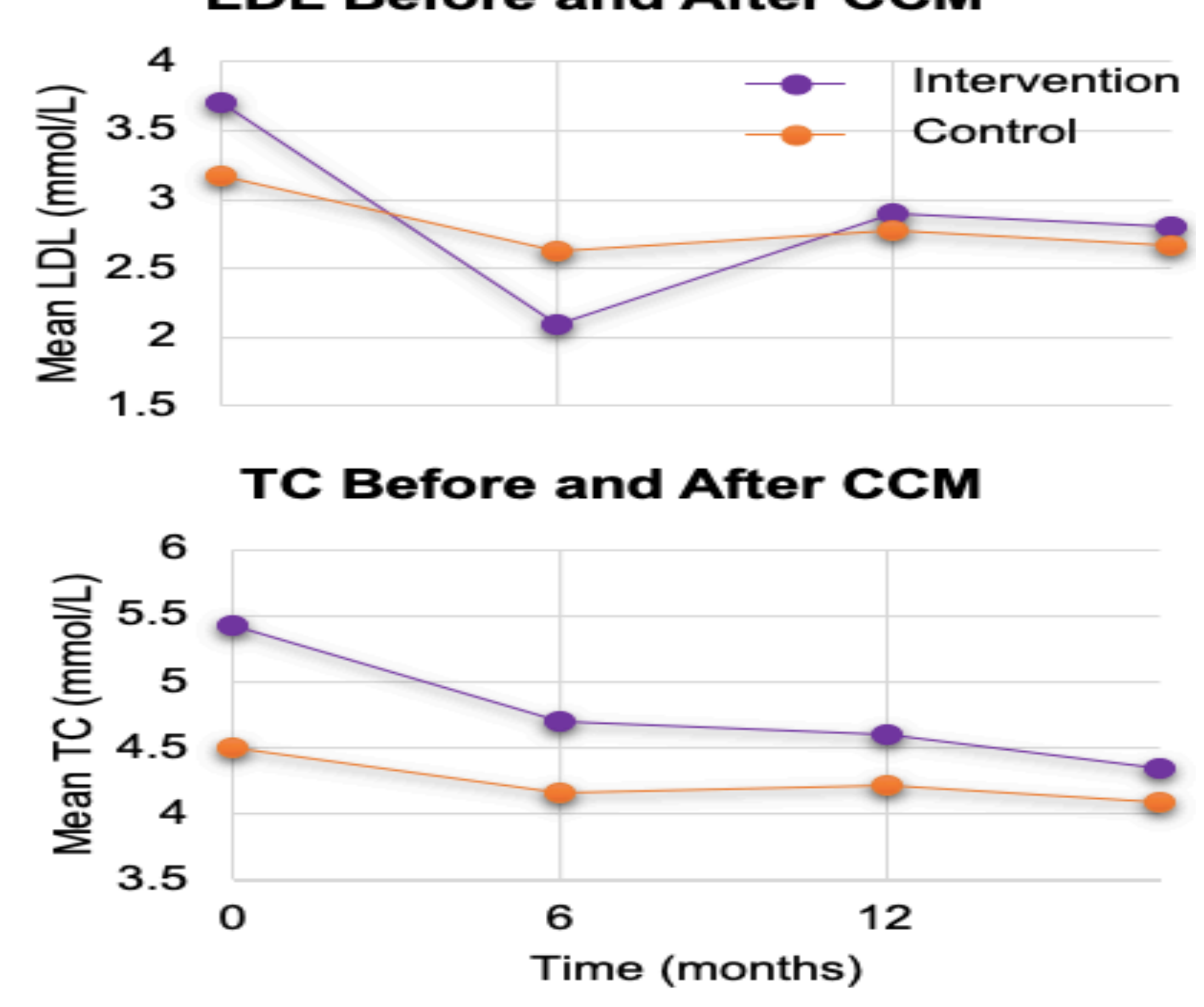

Figure 3. Lipid profile parameters

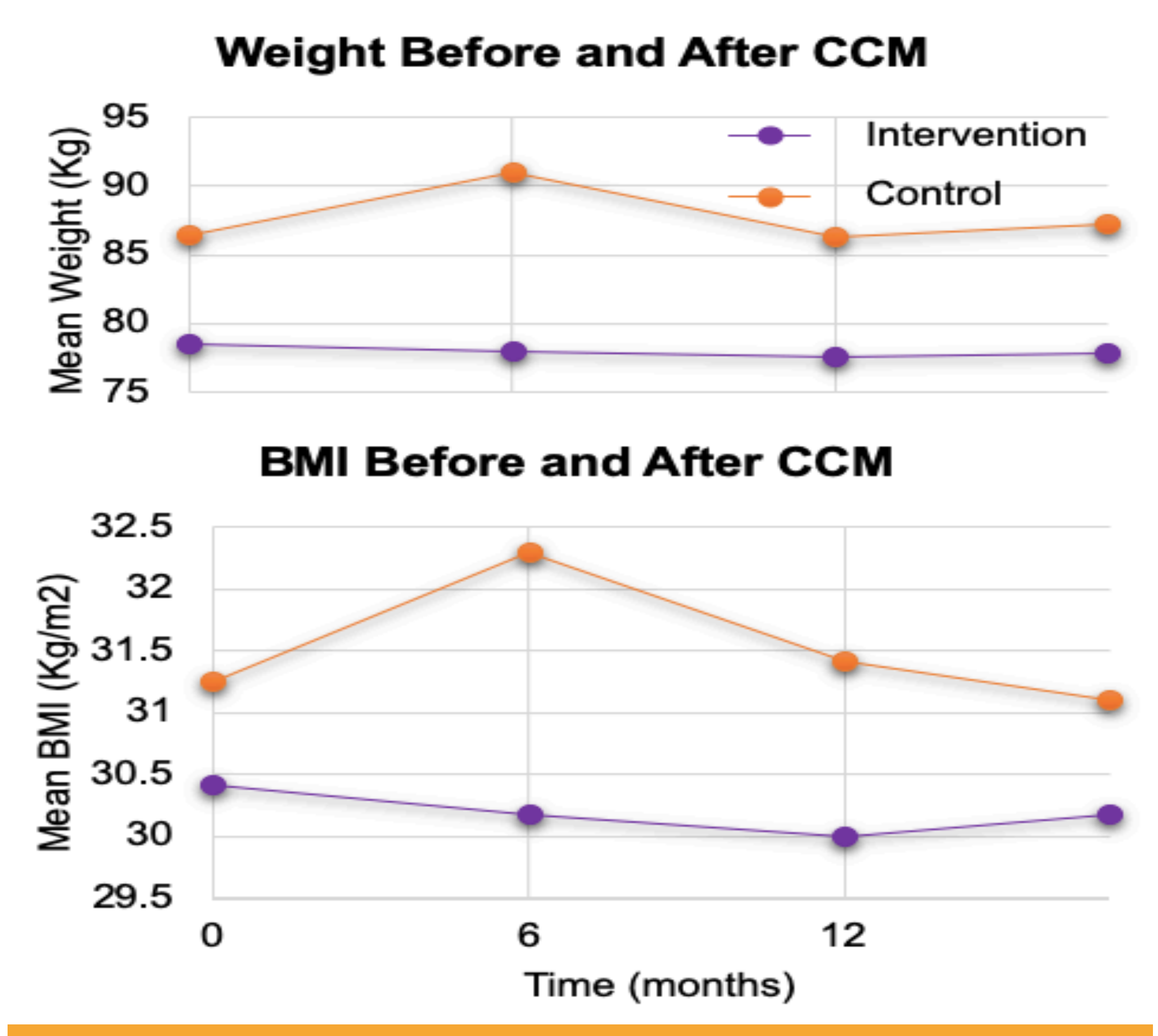

Figure 4. Anthropometric parameters

\section{Results (Cont'd)}

- There were no statistically significant differences at baseline or at 17 months in the medication regimens and types of medications

- The most commonly prescribed medication regimen was oral monotherapy.

- More patients in the intervention group received metformin than the control group at baseline and at 17months

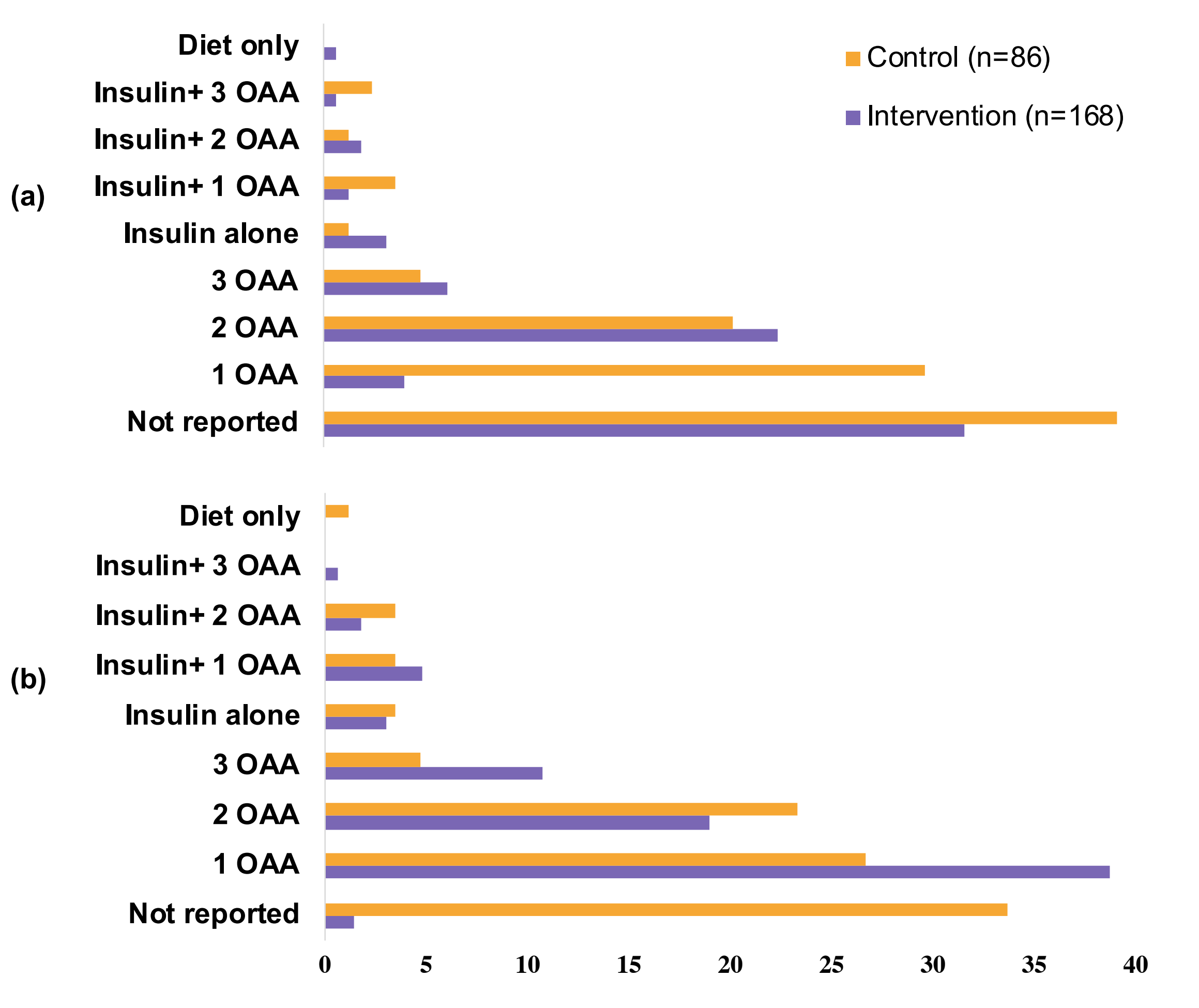

Figure 5. Medication regimens before and after CCM

\section{Limitations}

There is a high heterogeneity among the primary studies as a result of the variations in original studies setting, number of patients, type of pharmacist's interventions, and outcomes measures

- Each systematic review stratified the primary studies differently (based on study setting, disease, outcome, or not stratified)

\section{Conclusion}

CCM provision improved $\mathrm{HbA}_{1 \mathrm{c}}$, RBG, LDL-C, TC, weight, and BMI significantly in patients with diabetes within 17 month in a primary healthcare setting.

- Future studies should determine the long-term impact of CCM in this setting.

- The findings highlight the positive impact of the integration of different healthcare professionals into the healthcare team in primary care settings on tangible health outcomes.

\section{References}

Zimmet, P. Alberti, J Global and societal implications of the diabetes epidemic Nature. 414 (2001) 782-787. doi:10.1038/414782a.

2. Rhys Williams, S. Colagiuri, R. Almutairi, P.A. Montoya. IDF Diabetes Atlas ninth edition 2019, 2019. Global report on diabetes 2016. 1st ed. Geneva: World Health Organization. 2016.

3. D.R. Baptista, A. Wiens, R. Pontarolo, L. Regis, W.C.T. Reis, C.J. Correr, The chronic care model for type 2 diabetes: a systematic review, Diabetol. Metab. Syndr. 8 (2016) 1-7. doi:10.1186/s13098-015-0119-z.

J.H.V. Gilbert, J. Yan, S.J. Hoffman, Framework for action on interprofessional education and collaborative practice, J. Allied Health. 39 (2010) 196-197. 